\title{
Some spectral bounds for the harmonic matrix
}

\author{
Kinkar Ch. Das and Carlos M. da Fonseca
}

\begin{abstract}
The aim of this note is to establish new spectral bounds for the harmonic matrix.
\end{abstract}

The Harary matrix of given a connected graph $G$ of order $n$, say $R D(G)$, is an $n$-by- $n$ symmetric matrix, such that

$$
(R D(G))_{i j}=\left\{\begin{array}{cl}
\frac{1}{d_{i j}}, & \text { if } i<j, \\
0, & \text { if } i=j,
\end{array}\right.
$$

where $d_{i j}$ denotes the distance between the vertices $i$ and $j[10,11]$. This matrix (originally known as reciprocal distance matrix [11]) is particulary well-known in chemistry. This is mainly motivated by the importance of the influence of the neighbor atoms when compared with the more distance ones $[5,9,1]$.

If we consider a path of order $n$, with the vertices labeled in the standard way, the Harary matrix, say $A_{n}=\left(a_{i, j}\right)$, will be defined as

$$
a_{i, j}= \begin{cases}\frac{1}{|i-j|}, & \text { if } i \neq j, \\ 0, & \text { otherwise }\end{cases}
$$

Key Words: Reciprocal matrix, harmonic matrix, spectral bounds.

2010 Mathematics Subject Classification: Primary 15A18; Secondary 15B99, 05C50.

Received: 01.12.2016

Revised: 01.03.2017

Accepted: 06.03.2017 
which we will call harmonic matrix. The reason to adopt this name is intimately related with the harmonic numbers [13]. The $n$th harmonic number

$$
H_{n}=\sum_{k=1}^{n} \frac{1}{k}
$$

arises from the truncation of the harmonic series. One of the most interesting analytical expression for these numbers is

$$
H_{n}=\gamma+\Psi(n+1)
$$

where $\gamma$ is the Euler-Mascheroni constant and $\Psi(\cdot)$ is the digamma function. Clearly, we can extend this concept to

$$
H_{n}^{(r)}=\sum_{k=1}^{n} \frac{1}{k^{r}}
$$

which we may designate by $r$ th harmonic number, where $H_{n}^{(1)}=H_{n}$.

The harmonic spectrum of $A_{n}$ is

$$
\operatorname{Spec}_{H}\left(A_{n}\right)=\left\{\sigma_{1}, \sigma_{2}, \ldots, \sigma_{n}\right\},
$$

where $\sigma_{1} \geqslant \sigma_{2} \geqslant \cdots \geqslant \sigma_{n}$ are the eigenvalues of $A_{n}$, arranged in nonincreasing order. Sometimes we use $\sigma\left(=\sigma_{1}\right)$ to denote the largest eigenvalue of $A_{n}$. For example, the $5 \times 5$ harmonic matrix is

$$
A_{5}=\left(\begin{array}{ccccc}
0 & 1 & 1 / 2 & 1 / 3 & 1 / 4 \\
1 & 0 & 1 & 1 / 2 & 1 / 3 \\
1 / 2 & 1 & 0 & 1 & 1 / 2 \\
1 / 3 & 1 / 2 & 1 & 0 & 1 \\
1 / 4 & 1 / 3 & 1 / 2 & 1 & 0
\end{array}\right)
$$

The eigenvalues of $A_{5}$ are:

$$
2.61684,0.303284,-0.560813,-1.05328,-1.30603 \text {. }
$$

Another surprising application of the harmonic matrix is related to the study of the invertibility of the Foldy-Lax algebraic systems associated to both homogeneous and nonhomogeneous. The matrix commonly considered 
(cf. e.g. $[2,3,4])$ is

$$
B=\left(\begin{array}{ccccc}
\frac{1}{C_{1}} & \Phi_{\kappa}\left(z_{1}, z_{2}\right) & \Phi_{\kappa}\left(z_{1}, z_{3}\right) & \cdots & \Phi_{\kappa}\left(z_{1}, z_{n}\right) \\
\Phi_{\kappa}\left(z_{1}, z_{2}\right) & \frac{1}{C_{2}} & \Phi_{\kappa}\left(z_{2}, z_{3}\right) & \ddots & \vdots \\
\Phi_{\kappa}\left(z_{1}, z_{3}\right) & \Phi_{\kappa}\left(z_{2}, z_{3}\right) & \frac{1}{C_{3}} & \ddots & \Phi_{\kappa}\left(z_{n-2}, z_{n}\right) \\
\vdots & \ddots & \ddots & \ddots & \Phi_{\kappa}\left(z_{n-1}, z_{n}\right) \\
\Phi_{\kappa}\left(z_{1}, z_{n}\right) & \cdots & \Phi_{\kappa}\left(z_{n-2}, z_{n}\right) & \Phi_{\kappa}\left(z_{n-1}, z_{n}\right) & \frac{1}{C_{n}}
\end{array}\right),
$$

where the fundamental solution, $\Phi(x, y)$, of the Helmholtz equation in $\mathbb{R}^{3}$ with the fixed wave number $\kappa$ is given by

$$
\Phi(x, y)=\frac{e^{i \kappa|x-y|}}{4 \pi|x-y|}, \quad \text { for all } x, y \in \mathbb{R}^{3},
$$

and $C_{k}$ 's are coefficients based on surfaces area and impedance. There are some known sufficient conditions for the invertibility of $B$, but not necessary $[2,3,4]$. Inverting $B$ in general seems a hard problem. So, we normally want find a way to compute the inverse of $B$ as much generality as possible or eventually find necessary and sufficient conditions for its invertibility. If one considers the case where the distance between the centers of the consecutive scatterers is the same, i.e., distributing the scatterers in a uniform array, and the capacitances of the scatterers are also the same, i.e., the $C_{k}$ 's are equal, then $B$ is symmetric Toeplitz matrix, but not circulant. These considerations lead us, for $\kappa=0$, to the matrices of the form

$$
A_{n r}=\left(\begin{array}{ccccc}
0 & \frac{1}{r} & \frac{1}{2 r} & \cdots & \frac{1}{(n-1) r} \\
\frac{1}{r} & 0 & \frac{1}{r} & \ddots & \vdots \\
\frac{1}{2 r} & \frac{1}{r} & 0 & \ddots & \frac{1}{2 r} \\
\vdots & \ddots & \ddots & \ddots & \frac{1}{r} \\
\frac{1}{(n-1) r} & \cdots & \frac{1}{2 r} & \frac{1}{r} & 0
\end{array}\right)
$$

Finally, for $r=1$ we get the harmonic matrix (0.1).

Our aim is to establish several new results on the spectra of the harmonic matrices. It would be interesting to analyze in particular what happens in the case of the more general matrices (0.3).

\section{Known bounds}

In this section we present several known bounds applied to the harmonic matrix (0.1). The first theorem is a general result. 
Lemma 1.1. [8, Theorem 8.1.22] Let $A=\left(A_{i, j}\right)$ be an $n \times n$ irreducible nonnegative matrix with spectral radius $\sigma$ and let $R_{i}(A)=\sum_{j=1}^{n} a_{i, j}$ be the $i$ th row sum of $A$. Then

$$
\min \left\{R_{i}(A): 1 \leqslant i \leqslant n\right\} \leqslant \sigma \leqslant \max \left\{R_{i}(A): 1 \leqslant i \leqslant n\right\} .
$$

Moreover, if the row sums of $A$ are not all equal, then the both inequalities in (1.1) are strict.

For $A_{5}$, we have $2.08333<\sigma<3$.

From the bounds produced in [14] we have

$$
\sigma \leqslant \frac{3 n-4}{4}
$$

if $n$ is even, and

$$
\sigma \leqslant \frac{n-2+\sqrt{4 n^{2}-3}}{4}
$$

otherwise. For the case of $A_{5}$, the bound is 3.21221. From the same reference, we have

$$
\sigma \leqslant \frac{3 n-6+\sqrt{17 n^{2}+28 n+4}}{8}
$$

if $n$ is even, and

$$
\sigma \leqslant \frac{3 n-5+\sqrt{17 n^{2}+34 n-15}}{8},
$$

otherwise. For $A_{5}$, the bound is 4.2604 .

The bounds found by Zhou and Trinajstić in [15] give us

$$
\sqrt{\frac{\sum_{i=1}^{n} R_{i}^{2}}{n}}<\sigma<\max _{i} \sum_{j=1}^{n} h_{i j} \sqrt{\frac{R_{j}}{R_{i}}} .
$$

In the current example we get $2.59754<\sigma<2.77698$. Another bound from these authors is $3-\frac{4}{n}<\sigma$. Here, we have $2.2<\sigma$.

The first author in [6] established the bound

$$
\sigma \leqslant \frac{\sqrt{n^{2}+4 n-8}}{2} .
$$

For $n=5$, we get $\sigma<3.04138$. 


\section{New bounds}

We now give an upper bound for the spectral radius of the harmonic matrix $A_{n}$.

Theorem 2.1. For the harmonic matrix $A_{n}$, we have

$$
\sigma\left(A_{n}\right)< \begin{cases}\frac{n}{100}+8.356+\frac{2}{n} & \text { if } n \text { is even } \\ \frac{n}{100}+8.366 & \text { if } n \text { is odd }\end{cases}
$$

Proof. By Lemma 1.1, we have

$$
\sigma\left(A_{n}\right)< \begin{cases}2\left(1+\frac{1}{2}+\frac{1}{3}+\cdots+\frac{2}{n-2}\right)+\frac{2}{n} & \text { if } n \text { is even } \\ 2\left(1+\frac{1}{2}+\frac{1}{3}+\cdots+\frac{2}{n-1}\right) & \text { if } n \text { is odd }\end{cases}
$$

that is

$$
\sigma\left(A_{n}\right)< \begin{cases}2 H_{p-1}+\frac{1}{p} & \text { if } n=2 p \\ 2 H_{p} & \text { if } n=2 p+1 .\end{cases}
$$

One can easily get that

$$
1+\frac{1}{2}+\frac{1}{3}+\cdots+\frac{1}{100}<5.18738 .
$$

Using the above result, we have

$$
\begin{aligned}
2\left(1+\frac{1}{2}+\frac{1}{3}+\cdots+\frac{2}{n-1}\right)<2 & {\left[\left(1+\frac{1}{2}+\frac{1}{3}+\cdots+\frac{1}{100}\right)+\frac{1}{100} \times\left(\frac{n-1}{2}-100\right)\right] } \\
& <2\left[5.188+\frac{n-201}{200}\right] \\
& =\frac{n}{100}+8.366 .
\end{aligned}
$$

Similarly,

$$
2\left(1+\frac{1}{2}+\frac{1}{3}+\cdots+\frac{2}{n-1}\right)+\frac{2}{n}<\frac{n}{100}+8.356+\frac{2}{n} .
$$

This completes the proof. 
We now give a lower bound on $\sigma\left(A_{n}\right)$.

Theorem 2.2. Let $A_{n}$ be an $n \times n$ matrix defined in (0.1). Then

$$
\sigma\left(A_{n}\right) \geqslant \sqrt{\frac{2}{n} \sum_{i=1}^{n-1} \frac{n-i}{i^{2}}} .
$$

Proof. We have

$$
\begin{array}{rl}
\operatorname{tr}\left(A_{n}^{2}\right) & =2\left[n-1+\frac{n-2}{2^{2}}+\frac{n-3}{3^{2}}+\cdots+\frac{2}{(n-2)^{2}}+\frac{1}{(n-1)^{2}}\right] \\
=2 & n\left(1+\frac{1}{2^{2}}+\frac{1}{3^{2}}+\cdots+\frac{1}{(n-1)^{2}}\right)-2\left(1+\frac{1}{2}+\frac{1}{3}+\cdots+\frac{1}{(n-1)}\right) \\
& =2 \sum_{i=1}^{n-1} \frac{n-i}{i^{2}} .
\end{array}
$$

Therefore we have

$$
n \sigma^{2}\left(A_{n}\right) \geqslant \operatorname{tr}\left(A_{n}^{2}\right)=2 \sum_{i=1}^{n-1} \frac{n-i}{i^{2}}
$$

which gives the required result.

Lemma 2.3. Let $A_{n}$ be the harmonic matrix of order $n$. If $Y$ is a subset of $\{2,3, \ldots, n\}$, then

$$
\sum_{i \in Y} \sigma_{i}^{2}\left(A_{N}\right) \leqslant \frac{2(n-1)}{n} \sum_{i=1}^{n-1} \frac{n-i}{i^{2}}
$$

Proof. We have

$$
\sum_{i \in Y} \sigma_{i}^{2}\left(A_{n}\right) \leqslant \sum_{i=1}^{n} \sigma_{i}^{2}\left(A_{n}\right)-\sigma_{1}^{2}\left(A_{n}\right)
$$

Since

$$
\sum_{i=1}^{n} \sigma_{i}^{2}\left(A_{n}\right)=\operatorname{tr}\left(A_{n}^{2}\right)=2 \sum_{i=1}^{n-1} \frac{n-i}{i^{2}},
$$

by Theorem 2.2 with (2.3), we get the required result. 
Theorem 2.4. Let $A_{n}$ be the harmonic matrix of order $n$. If $\sigma_{k}\left(A_{n}\right) \leqslant 0$, for $2 \leqslant k \leqslant n$, then

$$
\left|\sigma_{k}\left(A_{n}\right)\right| \leqslant \sqrt{\frac{2(n-1)}{n(n-k+1)}} \sqrt{\sum_{i=1}^{n-1} \frac{n-i}{i^{2}}} .
$$

Proof. Since $\sigma\left(A_{n}\right) \geqslant \sigma_{2}\left(A_{n}\right) \geqslant \cdots \geqslant \sigma_{n}\left(A_{n}\right)$, one can see easily that

$(n-k+1) \sigma_{k}^{2}\left(A_{n}\right) \leqslant \sum_{i=k}^{n} \sigma_{i}^{2}\left(A_{n}\right) \leqslant \sum_{i=2}^{n} \sigma_{i}^{2}\left(A_{n}\right) \leqslant \frac{2(n-1)}{n} \sum_{i=1}^{n-1} \frac{n-i}{i^{2}}$, for $k \geqslant 2$.

Hence the theorem follows.

Corollary 2.5. Let $A_{n}$ be the harmonic matrix of order $n$. Then

$$
\left|\sigma_{n}\left(A_{n}\right)\right| \leqslant \sqrt{\frac{2(n-1)}{n}} \cdot \sqrt{\sum_{i=1}^{n-1} \frac{n-i}{i^{2}}} .
$$

Proof. By Theorem 2.4, we get the required result.

Gregory et al. [7, Theorem 2.1] obtained the spread of the spectrum of the adjacency matrix of graph $G$. Using the same technique, we give an upper bound for $\sigma-\sigma_{n}$. We omit its proof.

Theorem 2.6. Let $A_{n}$ be the harmonic matrix of order $n$ and precisely $k$ negative eigenvalues. Then

$$
\sigma-\sigma_{n} \leqslant\left(1+\frac{1}{k}\right) \sigma+\sqrt{2\left(1-\frac{1}{k}\right) \sum_{i=1}^{n-1} \frac{n-i}{i^{2}}-\left(1-\frac{1}{k^{2}}\right) \sigma^{2}} .
$$

Notice that all our news bounds are significantly better and useful for larger orders $n$.

\section{Acknowledgement.}

The first author was supported by the National Research Foundation of the Korean government with grant No. 2017R1D1A1B03028642. 


\section{References}

[1] T.S. Balaban, P.A. Filip, O. Ivanciuc, Computer generation of acyclic graphs based on local vertex invariants and topological indices. Derived canonical labelling and coding of trees and alkanes, J. Math. Chem. 11 (1992), 79-105.

[2] D.P. Challa, M. Sini, Multiscale analysis of the acoustic scattering by many scatterers of impedance type, Z. Angew. Math Phys. (2016), 67-58.

[3] D.P. Challa, M. Sini, The Foldy-Lax approximation of the scattered waves by many small bodies for the Lamé system, Math. Nachr. 288 (2015), 1834-1872.

[4] D.P. Challa, M. Sini, On the justification of the Foldy-Lax approximation for the acoustic scattering by small rigid bodies of arbitrary shapes, Multiscale Model. Simul. 12 (2014), 55-108.

[5] Z. Cui, B. Liu, On Harary matrix, Harary index and Harary energy, MATCH Commun. Math. Comput. Chem. 68 (2012), 815-823.

[6] K.C. Das, Maximum eigenvalue of the reciprocal distance matrix, J. Math. Chem. 47 (2010), 21-28.

[7] D.A. Gregory, D. Hershkowitz, S.J. Kirkland, The spread of the spectrum of a graph, Linear Algebra Appl. 332-334 (2001), 23-35.

[8] R.A. Horn, C.R. Johnson, Matrix Analysis, 2nd edition, Cambridge University Press, New York, 2013.

[9] O. Ivanciuc, T.S. Balaban, A.T. Balaban, Design of topological indices. Part 4. Reciprocal distance matrix, related local vertex invariants and topological indices, J. Math. Chem. 12 (1993), 309-318.

[10] O. Ivanciuc, T.S. Balaban, A.T. Balaban, Chemical graphs with degenerate topological indices based on information on distances, J. Math. Chem. 12 (1993), 21-33.

[11] D. Janežić, A. Miličević, S. Nikolić, N. Trinajstić, Graph Theoretical Matrices in Chemistry, Univ. Kragujevac, Kragujevac, 2007.

[12] D. Plavsić, S. Nikolić, N. Trinajstić, Z. Mihalić, On the Harary index for the characterization of chemical graphs, J. Math. Chem. 12 (1993), $235-250$. 
[13] J. Sondow, E.W. Weisstein, Harmonic Number, from MathWorld - A Wolfram Web Resource.

[14] L. Su, H. Li, M. Shi, J. Zhang, On spectral radius of the reciprocal distance matrix, Adv. Math., Beijing, 43 (2014), 551-558.

[15] B. Zhou, N. Trinajstić, Maximum eigenvalues of the reciprocal distance matrix and the reverse Wiener matrix, Int. J. Quantum Chem. 108 (2008), 858-864.

Kinkar Ch. Das,

Department of Mathematics,

Sungkyunkwan University,

Suwon 440-746, Republic of Korea.

Email: kinkardas2003@googlemail.com

Carlos M. da Fonseca,

Department of Mathematics,

Kuwait University,

Safat 13060, Kuwait.

Email: carlos@sci.kuniv.edu.kw

University of Primorska, FAMNIT,

Glagoljaška 8, 6000 Koper, Slovenia.

Email: carlos.dafonseca@famnit.upr.si 